\title{
A HYBRID SIMULATION MODELLING FRAMEWORK FOR COMBINING SYSTEM DYNAMICS AND AGENT-BASED MODELS
}

\author{
Ms. Le Khanh Ngan Nguyen \\ Department of Management Science \\ University of Strathclyde \\ Nguyen-le-khanh-ngan@strath.ac.uk
}

\author{
Prof Susan Howick \\ Department of Management Science \\ University of Strathclyde \\ Susan.howick@strath.ac.uk
}

\author{
Dr. Itamar Megiddo \\ Department of Management Science \\ University of Strathclyde \\ Itamar.megiddo@strath.ac.uk
}

\begin{abstract}
System dynamics (SD), discrete event simulation (DES) and agent-based model (ABM) are three different simulation modelling methods widely applied to support decision-making in complex systems across various disciplines. However, single simulation modelling approaches can face significant challenges representing the multi-dimensional nature of complex systems composed of interactive and interconnected constituents with dynamic behaviours. Combining different simulation methods offer an opportunity to overcome these challenges and to capture important characteristics and behaviours of such systems. Despite the growing interest and popularity in this approach, guidance for designing and utilizing hybrid models, especially for those combining SD and ABM, is scanty. This paper aims to review and consolidate the existing theoretical guidance/frameworks on combining these two simulation methods. Based on this literature review, we propose an initial framework for combining SD and ABM.
\end{abstract}

Keywords: System dynamics, agent-based model, hybrid simulation, theoretical frameworks.

\section{INTRODUCTION}

Although system dynamics (SD) and agent-based model (ABM) are different in terms of their philosophical approaches, both methods possess strong explanatory capabilities and can be combined and/or integrated (Bobashev et al, 2007; Phelan, 1999). The top-down approach of aggregated feedback of SD and the bottom-up approach of ABM may complement one another in a hybrid simulation modelling design to provide useful insights and realistic aspects of problems of complex systems. Combining SD and ABM enables problem owners to deal with different factors of system complexity, including micro, meso, and macro perspectives; strategic, tactical and operational levels; and detail and dynamic complexity (Begun et al, 2003; Morel \& Ramanujam, 1999). The scope of this paper is to consolidate the existing guidance on how to combine the two simulation modelling methods and propose a framework for developing hybrid SD-ABM models. 
Nguyen, Howick and Megiddo

\subsection{System Dynamics}

From an SD perspective, the interaction among the elements within a system and their interaction with the environment generate the characteristic behaviour of that system (Pidd, 1998). SD is a simulation modelling method which represents the structure of complex systems as accumulations (stocks), rates (flows), feedback and time delays, and examines their behaviour over time (Sterman, 2000). Stocks (or "levels") are defined as aggregation or accumulations of inflows and outflows over a period of time. Feedback exists when a change in a variable in the system impacts other variables in the system and these variables then, in turn, influence the initial variable. Delays represent the time it takes to measure and report information, make decisions or update stock that causes outputs to lag behind inputs.

\subsection{Agent-Based Model}

$\mathrm{ABM}$ is a simulation method for modelling autonomous, dynamic and adaptive systems and is formed on the basis of three key concepts which are agency, dynamics, and structure (Borshchev \& Filippov, 2004; Gunal, 2012). Agency means that agents are autonomous entities with specific properties, actions and possibly goals. Dynamics is the development, change, and evolvement of both agents and their environment over time. Structure is emergent as a result of agent interaction. Agents live in the environment, sense it and decide what action to employ at a certain time on the basis of the current state of the environment and their own state and defined decision rules. Agents can have explicit targets to minimize or maximize, and they can also learn and adapt based on their experiences. Such interactions result in the update of agents' internal state or decision on their next actions. The lower-level autonomy and interaction lead to the concept of dynamics at the system level. The system changes and patterns emerge as agents and their environment evolve or co-evolve over time. The core idea of ABM is that a model composed of agents that interact with one another and their environment can effectively demonstrate many (if not most) phenomena and real-world systems (Wilensky \& Rand, 2015).

\section{THE EXISTING THEORETICAL GUIDANCE ON COMBINING SD AND ABM}

$\mathrm{SD}$ and $\mathrm{ABM}$ have already been used separately to study the same problem in some areas which has led to interesting outcomes. For example, Scholl (2001) compared SD and ABM literature on the bullwhip phenomenon, which arises in supply-chain management, and Rahmandad and Sterman (2008) studied literature using these methodologies to model "networking" problems such as innovation diffusion and AIDS dissemination through needle sharing (Rahmandad \& Sterman, 2008; Scholl, 2001). These reviews indicate differences and similarities between results and explanations of the studied phenomena in the two simulation modelling methods. In addition to supply chain management and diffusion, SD and ABM methods were also compared in areas such as ecology (Norling, 2007) and biology (Wakeland et al, 2004). Applying the two methods separately to study the same problem provide fruitful insights, crossvalidation, and triangulation of results (Phelan, 2004). While the early works focus on the use of one simulation modelling method to validate outputs generated by the other and triangulate outputs, a growing number of studies using hybrid SD-ABM approaches have shown the diversity in the designs of hybridization of the two methods. We conducted a review of literature on the existing theoretical guidance to design a hybrid simulation model that combines SD and ABM and summarized the results of different designs for a hybrid SD-ABM model in Table 1. Although some of the studies included in this table provide guidance on mixing SD and discrete event simulation (DES), mixing analytic and simulations modelling, or mixing methods in general, the hybrid designs they proposed can be used for mixing SD and ABM. It should be noted that we did not include Lättilä et al (2010), Onggo (2014), and 
Djanatliev and German (2015) in Table 1. Although these studies provide guidance on mixing simulation methods, they did not provide a description of specific hybrid designs.

We identified and classified the existing combinations of SD and ABM into six designs. As the literature uses different sets of terminology to describe similar designs, we will not explain all terminology but only the general ideas for each design. Detailed explanations can be found in the referenced papers. When using a parallel design, SD and ABM are used to develop independent models either to address different aspects of the same problem which are better suited with one particular simulation method or to represent the same problem for direct comparison. Results of these models are ultimately combined to solve the same problem or compared to enhance confidence in output produced by each model. A sequential design includes two or more separate sub-models embedded in different simulation modelling methods in which one model is used to inform the other. One simulation is initially run, and it produces output before terminating; the second simulation starts to run, using as input the output of the first simulation. The information and/or data are passed only once from the first to the second simulation. The output of the second simulation represents the final output of the hybrid model. An interaction design comprises different sub-models developed using different simulation modelling approaches which are considered equally important and interact cyclically during run time. Interactions between sub-models occur several times in each direction. A sequential design can be considered as a special case of the interaction design when the interaction occurs once and in one direction only. Integration is an approach that combines different simulation modelling methods to create one seamless hybrid model in which it is impossible to explicitly distinguish between the SD and ABM parts and to identify where one simulation approach ends and the other begins. This design offers a coherent view of the problem which enhances continuous flows of information and feedback and captures interactive effects within a system. Although several studies concur on the definition of an integration design, only Swinerd and McNaught (2012 \& 2014) describe in detail different ways to develop an integrated hybrid model. They proposed three designs which belong to the integrated class, including agents with rich internal structure, stocked agents, and parameters with emergent behaviour. This design combines different simulation modelling methods to form one unified hybrid model in which one method dominates and is enhanced by elements of another. As enrichment and integration designs share many similarities, there seems to be a continuum from enrichment to full integration in hybrid simulation modelling designs depending on the relative dominance between the adopted simulation approaches. An enrichment design uses an element of one simulation method to enhance the main method without the need to build an additional model, while integration brings together two full methods to create something new. A dynamic design allows the dynamic switching between SD and ABM in the structure of a model. Its reported application is to efficiently depict the process of an ongoing epidemic. The extent to which sub-models in a hybrid simulation model are coupled depends on its design and are increasingly coupled in the following order: parallel (genuinely independent), sequential (loosely coupled), interaction, dynamic, enrichment, and integration (inseparably coupled).

Table 1 The existing theoretical guidancelframeworks for combining SD and ABM

\begin{tabular}{|l|l|l|l|l|l|l|}
\hline \multirow{2}{*}{ References } & \multicolumn{5}{|c|}{ Designs for a hybrid SD-ABM model } \\
\cline { 2 - 6 } & Parallel & Sequential & Interaction & Enrichment & \multicolumn{1}{|c|}{ Integration } & Dynamic \\
\hline $\begin{array}{l}\text { (Shanthikumar } \begin{array}{l}\text { \& Sargent, } \\
1983)\end{array} \\
\text { (Bennett, 1985) }\end{array}$ & $\begin{array}{l}\text { Class I } \\
\text { on }\end{array}$ & Class III, IV & & Class II & \\
\hline $\begin{array}{l}\text { (Kim \& Juhn, } \\
\text { 1997) }\end{array}$ & & & Enrichment & Integration & \\
& & & $\begin{array}{l}\text { Multi-Agent Dynamics where a } \\
\text { hybrid model is constructed with } \\
\text { the principles of SD and using } \\
\text { array variables to represent the } \\
\text { individual agents }\end{array}$ & \\
\hline
\end{tabular}




\begin{tabular}{|c|c|c|c|c|c|c|}
\hline $\begin{array}{l}\text { (Parunak et al, } \\
\text { 1998) }\end{array}$ & & & & \multicolumn{2}{|c|}{$\begin{array}{l}\text { Agents modeled using the } \\
\text { equations of SD. } \\
\text { An agent can be part of a bigger } \\
\text { SD. }\end{array}$} & \\
\hline $\begin{array}{l}\text { (Akkermans, } \\
\text { 2001) }\end{array}$ & & & & \multicolumn{2}{|c|}{$\begin{array}{l}\text { Using SD to model the logic of } \\
\text { individual agents. }\end{array}$} & \\
\hline $\begin{array}{l}\text { (Schieritz \& } \\
\text { GroBler, 2003) }\end{array}$ & & & & \multicolumn{2}{|c|}{$\begin{array}{l}\text { Using SD to model the internal } \\
\text { decision logic or cognitive } \\
\text { structure of the agents in an } \\
\text { ABM. }\end{array}$} & \\
\hline $\begin{array}{l}\text { (Borshchev \& } \\
\text { Filippov, 2004) }\end{array}$ & & & & \multicolumn{2}{|c|}{$\begin{array}{l}\text { SD sub-models inside discretely } \\
\text { communicating agents. } \\
\text { Agents live in an environment } \\
\text { whose dynamics is modeled using } \\
\text { SD. }\end{array}$} & \\
\hline $\begin{array}{l}\text { (Lorenz \& Jost, } \\
2006)\end{array}$ & & & & \multicolumn{2}{|c|}{$\begin{array}{l}\text { Using SD structures to create } \\
\text { entities for an ABM. } \\
\text { An "active" environment }\end{array}$} & \\
\hline $\begin{array}{l}\text { (Bobashev et al, } \\
\text { 2007) }\end{array}$ & & & & & & $\begin{array}{l}\text { Hybrid } \\
\text { threshold } \\
\text { model }\end{array}$ \\
\hline $\begin{array}{l}\text { (Martinez- } \\
\text { Moyano et al, } \\
\text { 2007) }\end{array}$ & & $\begin{array}{l}\text { Scenario } \\
\text { exploration } \\
\text { and Crisis } \\
\text { response }\end{array}$ & & & $\begin{array}{l}\text { Intertwined } \\
\text { models }\end{array}$ & \\
\hline $\begin{array}{l}\text { (Chahal \& } \\
\text { Eldabi, 2008) }\end{array}$ & & & $\begin{array}{l}\text { Hierarchical } \\
\text { format }\end{array}$ & $\begin{array}{l}\text { Process - } \\
\text { Environment } \\
\text { format }\end{array}$ & $\begin{array}{l}\text { Integration } \\
\text { format }\end{array}$ & \\
\hline $\begin{array}{l}\text { (Brailsford et } \\
\text { al, 2010) }\end{array}$ & & & & & $\begin{array}{l}\text { The "Holy } \\
\text { Grail" }\end{array}$ & \\
\hline $\begin{array}{l}\text { (Vincenot et al, } \\
2011 \text { ) }\end{array}$ & & & & \multicolumn{2}{|c|}{ Case 1,2 , and 3} & Case 4 \\
\hline $\begin{array}{l}\text { (Swinerd \& } \\
\text { McNaught, } \\
\text { 2012, 2014) }\end{array}$ & $\begin{array}{l}\text { Interfaced } \\
\text { class }\end{array}$ & $\begin{array}{l}\text { Sequential } \\
\text { class }\end{array}$ & & & $\begin{array}{l}\text { Integrated class } \\
\text { including: } \\
\text { Agents with rich } \\
\text { internal structure, } \\
\text { stocked agents, } \\
\text { parameters with } \\
\text { emergent } \\
\text { behaviour }\end{array}$ & \\
\hline $\begin{array}{l}\text { (Chahal et al, } \\
\text { 2013) }\end{array}$ & & $\begin{array}{l}\text { Cyclic } \\
\text { interaction }\end{array}$ & $\begin{array}{l}\text { Parallel } \\
\text { interactions }\end{array}$ & & & \\
\hline $\begin{array}{l}\text { (Wallentin \& } \\
\text { Neuwirth, } \\
\text { 2017) }\end{array}$ & & & & & "Super-agents" & $\begin{array}{l}\text { Dynamica } \\
\text { lly } \\
\text { switching } \\
\text { hybrid } \\
\text { model }\end{array}$ \\
\hline $\begin{array}{l}\text { (Morgan et al, } \\
\text { 2017) }\end{array}$ & Parallel & Sequential & Interaction & Enrichment & Integration & \\
\hline
\end{tabular}

\section{LIMITATIONS OF EXISTING GUIDANCE FOR HYBRIDIZING SD AND ABM}

There are three major limitations of the studies shown in table 1 when providing guidance on combining SD and ABM Firstly, they do not specify the processes that modellers need to take and which aspects they need to consider to reach a decision on the design of a hybrid model. Secondly, we note that such 
guidance is established at a high level and it is, therefore, still quite abstract and not straightforward for problem owners to apply in solving a specific problem. Lastly, most of the existing hybrid simulation modelling studies focus on dealing with issues of particular domains such as inter-organizational network development in Akkermans (2001) or supply chain management in Schieritz and Größler (2003), rather than offering a broader but more detailed guidance specifying when, why, and how to combine SD and ABM approaches. In this paper we focus on describing "how" the two methods can interact and exchange data and information. The paper seeks to provide a detailed and practical stepwise instruction that specifies what steps modellers need to take and what they need to do in each step to build a hybrid simulation model. This is achieved through building on the existing guidance on hybrid SD-ABM modelling and reflecting on existing hybrid SD-ABM studies and the process of building a hybrid model. A guideline presented in a consistent and structured format will assist the selection of appropriate model designs in future studies, which will further facilitate and enhance the efficiency of the process of developing a hybrid model and the usefulness of the created models.

\section{A HYBRID SD-ABM SIMULATION MODELLING FRAMEWORK}

We propose a hybrid SD-ABM simulation modelling framework that aids modellers in the conceptual design phase of the development of a hybrid model. We expand and elaborate the existing work in this research area and focus on the processes that are essential to structure the problems into hybrid simulation models of which discussing different designs of hybridization is one part. In the proposed framework, the iterative process of developing a hybrid simulation model has been divided into 9 steps. The first three steps aim to specify the characteristics of the problem of interest on which modellers determine whether an individual SD or ABM or a hybrid SD-ABM is most suited to modelling the problem. In the fourth step, modellers determine different modules within a hybrid model, the hierarchy, and levels of abstraction for each of them. A simulation modelling method is chosen for each module in the fifth step. After defining the flows of information among modules in the sixth step, in the next step, the modeller decides on a design to combine these modules (i.e., combing different simulation modelling methods into a hybrid model). Finally, interfaces between modules and updating rules are defined.

\subsection{Conceptualizing the Problem}

Before developing a simulation model, it is vital to be clear about the nature of a problem under investigation and the objectives of the model. This helps to identify the scope of the model and the level of detail required and, therefore, the choice of appropriate simulation modelling methods (Roberts et al, 2012; Robinson, 2008). In addition to reviewing literature describing the problem and existing models addressing related problems, modellers should widely consult with relevant stakeholders and experts to refine the problem definition and develop clear, agreed modelling objectives. Defining the objectives of the model is an iterative process as deepening understanding of the problem gained from a modelling process may alter objectives. A more detailed understanding of the problem also guides modelling decisions. For example, building a simulation model to guide a healthcare practice or a public health policy should carefully and explicitly define the problem characteristics such as the target population, the healthcare setting, the cost of different interventions and how it can be modelled, the health outcomes of importance for that population, and the time horizon adequate to capture differences in outcome across interventions.

\subsection{A Problem-Oriented Approach to Choosing Between a Single and Hybrid Simulation Modelling Method}

Once the problem is conceptualized, it is important to identify whether the problem of interest can be modeled and solved using a single simulation modelling method or requires a hybrid simulation approach. Each simulation modelling method has strengths and limitations, making it better suited for specific 
problems and less so for other ones (Scholl, 2001). The selection of different simulation modelling methods should, therefore, be driven by the problem characteristics. Lättilä, et al (2010) has listed some different "problematic situations" where one of the simulation modelling methods is preferred to use. This helps answer the questions on why and when it is appropriate to use hybrid simulation models. Modellers will choose a hybrid simulation modelling approach that combines the strengths of SD and ABM if one simulation paradigm has difficulty to capture the complexity of the problem on its own.

\subsection{Determining Modules, Hierarchy, and Levels of Abstraction}

A model can consist of several components called "modules". A module should principally be selfcontained and bounded with predefined interfaces (input and output) to the external world, including other modules. In a hybrid simulation model, we find it useful to consider a module as one logical component of a hybrid model developed using one of the simulation modelling methods (Onggo, 2014). In an integrated hybrid model, the boundary between modules is not explicit because the interfaces between modules are intertwined. In this case, we still can dissect the model into smaller components, where each component will be considered as a module that can receive a set of inputs and transform them into a set of outputs. Djanatliev and German's 2015 work aligns with this idea; they raised the necessity to define independent problem areas within a specific domain scope and to model each area using one of the simulation methods (Djanatliev \& German, 2015). There are several options to perform this task. For example, in dealing with the problems in healthcare we can explore the problems in different healthcare settings such as hospitals and long-term care facilities. We can also use a hierarchical breakdown to study the problems in healthcare at a national/regional level (macro), an institutional level (meso/micro) and an individual level (micro).

\subsection{Selecting Simulation Modelling Methods for Each Module}

This step concurs with Horizontal Paradigm Linking proposed by Djanatliev and German (2015). After identifying the modules of a problem, modellers need to justify the selection of a particular simulation modelling method used for each module and whether it is the most appropriate for the job (Brailsford et al, 2013). Specifying the modelling hierarchy in a hybrid SD-ABM model, which is the hierarchical level of an SD module relative to that of an ABM module, also aids the choice of a simulation method for each module. Figure 1 represents different types of hierarchy levels for different designs of hybrid SD-ABM simulation modelling.

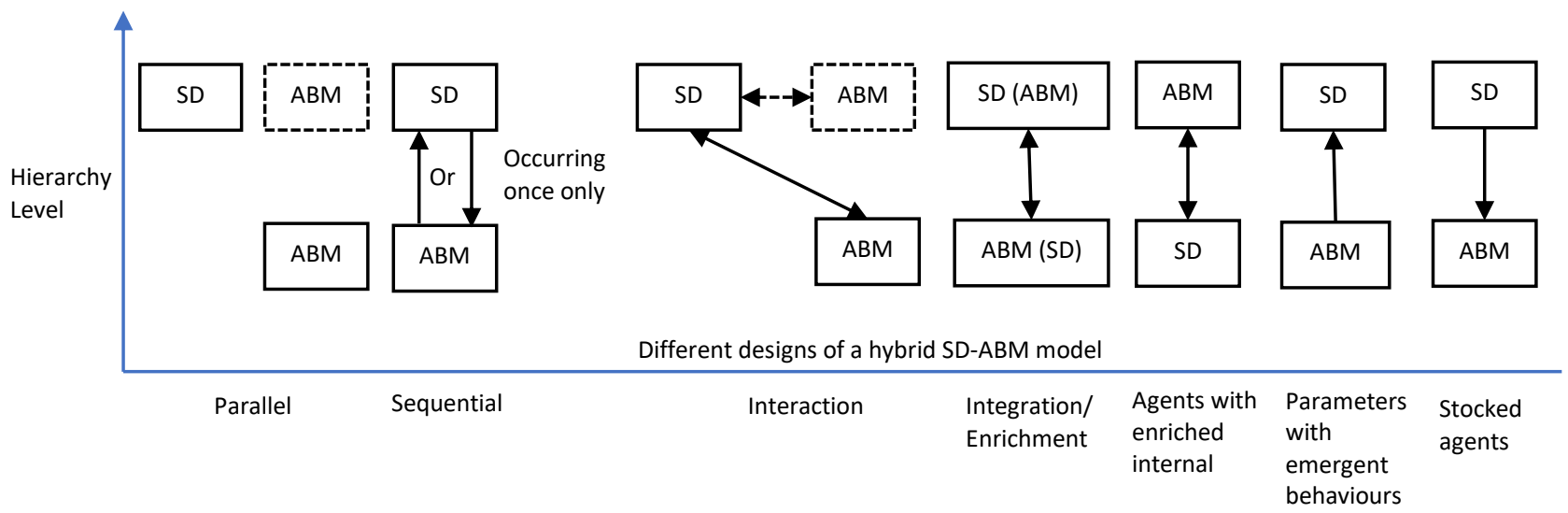

Figure 1 The hierarchy levels and information flows for different designs of SD-ABM hybrid models 


\subsection{Defining the Flow of Information between Modules}

In this step, modellers decide on the paths and directions of information flow between modules in a hybrid model. Figure 1 describes the flow paths and directions of information between an SD module and an ABM module in a hybrid model by arrows. Single-headed arrows indicate that information only flows in one direction while double-headed arrows show an exchange of information in both directions between two modules. Boxes with dashed borders indicate that the hierarchical level of a module can be at any level but not occupying all levels simultaneously. In the sequential design, information is passed in one direction, either from an SD module to an ABM module or vice versa. Decisions on the flow of information between modules will support the modeller's choice of the most appropriate design for a hybrid simulation model.

\subsection{Selecting a Hybrid Simulation Modelling Design}

Defining the flows of information and choosing a hybrid simulation design are in essence Vertical Paradigm Linking, previously described by Djanatliev and German (2015). The flows of data from an $\mathrm{ABM}$ module to an SD module can be communicated in several different manners: sending the total number of agents with specific attributes in the ABM as an inflow to the SD module; the emergent property of an ABM influencing the relationship governing a stock level in the SD module; sending a population size based on an SD module stock to an ABM module within a predefined unit of time, and individual agents can be generated using distribution functions based on existing empirical data or theories to represent the necessary heterogeneity of these agents; and using the size of a stock in an SD module to affect agents' behaviours and goals as well as their environment's attributes. The hierarchy of modules and how information exchanges between them inform the selection of a design for hybridization as shown in Figure 1. We will discuss the detailed description of each hybrid SD-ABM design and examples of its application to explore the specific problems of healthcare associated infections in later work. Figure 2 shows how the design of a hybrid simulation model is chosen step by step.

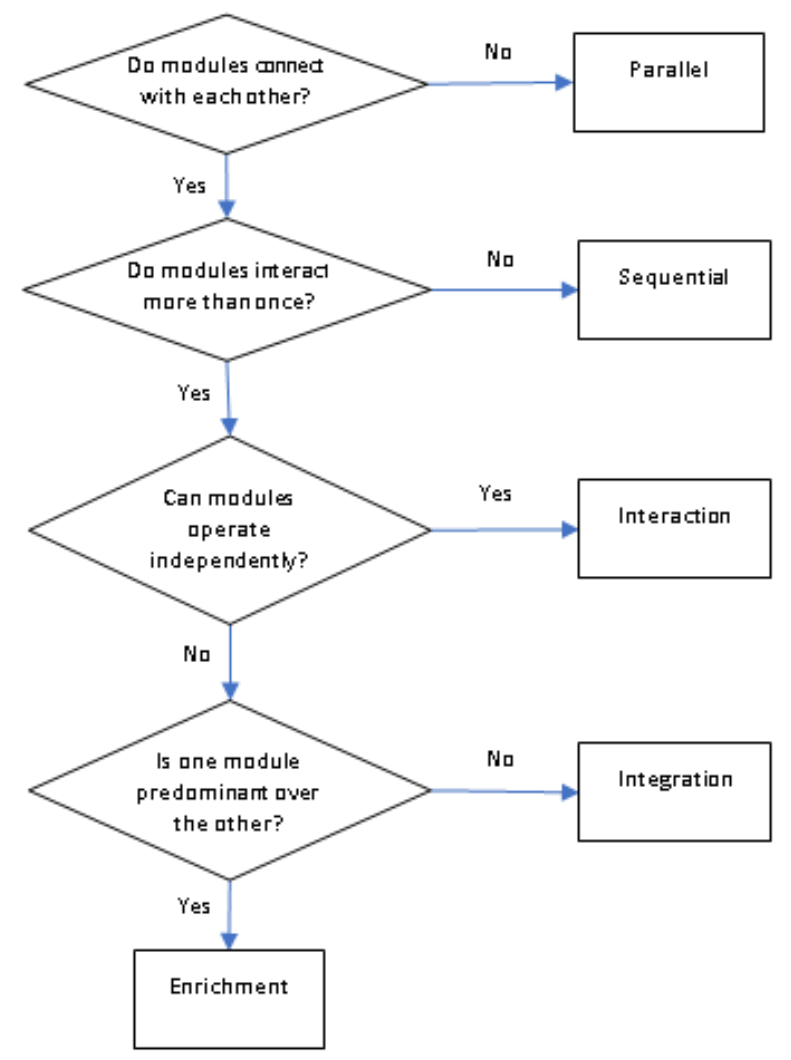

Figure 2 Selection of a hybrid simulation modelling design 
Nguyen, Howick and Megiddo

\subsection{Defining Interfaces}

In the second last step, the modeller identifies clear and logical interfaces between modules. An interface that decouples the two modules defines the information passing from one module to the other, the module generating the information and the one receiving the information during the running time of the hybrid model. It is also important to determine how the output produced by a module is treated: whether the output information will become input for another module, form a part or the entire output for the hybrid model, or both. As one module in a hybrid model is represented by SD while another module is implemented in ABM, they have different levels of details. The information needs to be aggregated when moving from a lower level of detail to a higher level and disaggregated when moving in the reverse direction.

\subsection{Defining Updating Rules}

Updating rules will define when the information will be sent from one module to another and how new information is handled by the receiving module to maintain the logical consistency of the whole hybrid model (Onggo, 2014). Modellers also need to consider the running time of a model when defining updating rules. Although the modules in a hybrid SD-ABM model use the same time advancement method, namely fixed-time increments, they may use different time units. Additionally, modules can be run on different simulation modelling software which has its own internal time management. If SD and ABM modules use the same unit of time, updates can be easily done when the hybrid model advances its simulation time. However, this may slow down simulation run time if one runs faster than the other for same simulated time unit. One module will have to wait for the other to finish. This will be a bigger problem in large models such as models with many agents. If the modules use different units of time, updates can occur asynchronously or synchronously. Asynchronously, every time a module advances its simulation time, the module's status may alter and it will send new information to recipient modules which the interfaces define (Onggo, 2014). Synchronously, all modules in a hybrid model will pass their information to other recipient modules at predefined simulation points which can be, for example, the time step of one of the modules.

\section{CONCLUSION}

This proposed framework is still in its infancy but considered as a good starting point to build up a more comprehensive version as the research evolves. In future work, we will apply this framework to design a hybrid SD-ABM model to explore a problem in healthcare-associated infection prevention and control. Based on the experience from implementing the framework to build the model, we also reflect upon the framework by considering what was necessary and appropriate to facilitate the process of modelling, what was not applicable and what changes should be made to enhance the practicality of the framework.

\section{REFERENCES}

Akkermans, H. (2001). Renga: a systems approach to facilitating inter-organizational network development. System Dynamics Review, 17(3), 179-193. doi:10.1002/sdr.215

Begun, J. W., Zimmerman, B., \& Dooley, K. (2003). Health care organizations as complex adaptive systems. Advances Health Care Organization Theory, 253, 253-288.

Bennett, P. G. (1985). On Linking Approaches to Decision-Aiding: Issues and Prospects. The Journal of the Operational Research Society, 36(8), 659-669. doi:10.2307/2582261

Bobashev, G. V., Goedecke, D. M., Feng, Y., \& Epstein, J. M. (2007, December). A Hybrid Epidemic Model: Combining The Advantages Of Agent-Based And Equation-Based Approaches. Paper presented at the 2007 Winter Simulation Conference.

Borshchev, A., \& Filippov, A. (2004). From System Dynamics and Discrete Event to Practical Agent Based Modeling: Reasons, Techniques, Tools. Paper presented at the Proceedings of the 22nd international conference of the system dynamics society, Oxford. 
Brailsford, S., Desai, S., \& Viana, J. (2010). Towards the Holy Grail: combining system dynamics and discrete-event simulation in healthcare. (Vol. 2293-2303).

Brailsford, S., Viana, J., Rossiter, S., Channon, A. A., \& Lotery, A. J. (2013, 8-11 Dec). Hybrid simulation for health and social care: The way forward, or more trouble than it's worth? Paper presented at the Proceedings of the 2013 Winter Simulation Conference: Simulation: Making Decisions in a Complex World.

Chahal, K., \& Eldabi, T. (2008, 7-10 Dec). Applicability of hybrid simulation to different modes of governance in UK healthcare. Paper presented at the Winter Simulation Conference.

Chahal, K., Eldabi, T., \& Young, T. (2013). A conceptual framework for hybrid system dynamics and discrete event simulation for healthcare. Journal of Enterprise Information Management, 26(1/2), 50-74.

Djanatliev, A., \& German, R. (2015, 6-9 Dec). Towards a guide to domain-specific hybrid simulation. Paper presented at the 2015 Winter Simulation Conference (WSC).

Gunal, M. M. (2012). A guide for building hospital simulation models. Health Systems, 1(1), 17-25. doi:10.1057/hs.2012.8

Kim, D.-H., \& Juhn, J.-H. (1997). System Dynamics as a Modeling Platform for Multi-Agent Systems. Paper presented at the The 15th International Conference of the System Dynamics Society Istanbul, Turkey.

Lorenz, T., \& Jost, A. (2006). Towards an orientation framework in multi-paradigm modeling. Paper presented at the Proceedings of the 24th International Conference of the System Dynamics society.

Martinez-Moyano, I., Sallach, D., Bragen, M., \& Thimmapuram, P. R. (2007). Design for a Multilayer Model of Financial Stability: Exploring the Integration of System Dynamics and Agent-based Models.

Morel, B., \& Ramanujam, R. (1999). Through the Looking Glass of Complexity: The Dynamics of Organizations as Adaptive and Evolving Systems. Organization Science, 10(3), 278-293. doi:10.1287/orsc.10.3.278

Morgan, J. S., Howick, S., \& Belton, V. (2017). A toolkit of designs for mixing Discrete Event Simulation and System Dynamics. European Journal of Operational Research, 257(3), 907-918. doi:https://doi.org/10.1016/j.ejor.2016.08.016

Norling, E. (2007). Contrasting a System Dynamics Model and an Agent-Based Model of Food Web Evolution. Paper presented at the Multi-Agent-Based Simulation VII, Berlin, Heidelberg.

Onggo, B. S. (2014, 7-10 Dec). Elements of a hybrid simulation model: A case study of the blood supply chain in low- and middle-income countries. Paper presented at the Proceedings of the Winter Simulation Conference

Parunak, H. V. D., Savit, R., \& Riolo, R. L. (1998). Agent-based modeling vs. equation-based modeling: A case study and users' guide. Paper presented at the International Workshop on Multi-Agent Systems and Agent-Based Simulation.

Phelan, S. E. (1999). A Note on the Correspondence Between Complexity and Systems Theory. Systemic Practice and Action Research, 12(3), 237-246. doi:10.1023/A:1022495500485

Phelan, S. E. (2004). Using integrated top-down and bottom-up dynamic modeling for triangulation and interdisciplinary theory integration. Paper presented at the The 22nd international conference of the System Dynamics Society, Oxford.

Pidd, M. (1998). Computer simulation in management science (Vol. 4): Wiley Chichester.

Rahmandad, H., \& Sterman, J. (2008). Heterogeneity and Network Structure in the Dynamics of Diffusion: Comparing Agent-Based and Differential Equation Models. Management Science, 54(5), 998-1014. doi:10.1287/mnsc.1070.0787

Roberts, M., Russell, L. B., Paltiel, A. D., Chambers, M., McEwan, P., \& Krahn, M. (2012). Conceptualizing a model: a report of the ISPOR-SMDM modeling good research practices task force-2. Medical Decision Making, 32(5), 678-689. 
Robinson, S. (2008). Conceptual modelling for simulation Part I: definition and requirements. Journal of the Operational Research Society, 59(3), 278-290. doi:10.1057/palgrave.jors.2602368

Schieritz, N., \& GroBler, A. (2003, 6-9 Jan). Emergent structures in supply chains - a study integrating agent-based and system dynamics modeling. Paper presented at the 36th Annual Hawaii International Conference on System Sciences.

Scholl, H. J. (2001, July 23-27). Looking across the fence: Comparing findings from SD modeling efforts with those of other modeling techniques. Paper presented at the In Proceedings of the 19th international conference of the system dynamics society, Atlanta, USA.

Shanthikumar, J. G., \& Sargent, R. G. (1983). A Unifying View of Hybrid Simulation/Analytic Models and Modeling. Operations Research, 31(6), 1030-1052. doi:10.1287/opre.31.6.1030

Sterman, J. (2000). Business Dynamics, System Thinking and Modeling for a Complex World. (Vol. 19).

Swinerd, C., \& McNaught, K. R. (2012). Design classes for hybrid simulations involving agent-based and system dynamics models. Simulation Modelling Practice and Theory, 25, 118-133.

Swinerd, C., \& McNaught, K. R. (2014). Simulating the diffusion of technological innovation with an integrated hybrid agent-based system dynamics model. Journal of Simulation, 8(3), 231-240.

Vincenot, C. E., Giannino, F., Rietkerk, M., Moriya, K., \& Mazzoleni, S. (2011). Theoretical considerations on the combined use of system dynamics and individual-based modeling in ecology. Ecological Modelling, 222(1), 210-218.

Wakeland, W., Gallaher, E., Macovsky, L., \& Aktipis, C. (2004). A comparison of system dynamics and agent-based simulation applied to the study of cellular receptor dynamics.

Wallentin, G., \& Neuwirth, C. (2017). Dynamic hybrid modelling: Switching between AB and SD designs of a predator-prey model. Ecological Modelling, 345, 165-175. doi:https://doi.org/10.1016/j.ecolmodel.2016.11.007

Wilensky, U., \& Rand, W. (2015). An Introduction to Agent-Based Modeling: Modeling Natural, Social, and Engineered Complex Systems with NetLogo: The MIT Press.

\section{AUTHOR BIOGRAPHIES}

LE KHANH NGAN NGUYEN is a PhD student at the Department of Management Science at the University of Strathclyde. She received both a Bachelor of Pharmacy and Master of Public Health from the University of Sydney. Her research topic is hybrid health systems simulation modelling in controlling healthcare-associated infections.

SUSAN HOWICK is a Professor of Management Science. She gained a BSc in Mathematics from Dundee University and a MSc in Operational Research and a $\mathrm{PhD}$ in Management Science from Strathclyde. Her main research interest lies in taking a systems perspective when using models to support decision-makers. She is also interested in exploring approaches to integrating models that promote client value from the modelling process. Susan is currently a Vice-President of the Operational Research Society and was President of the Policy Council for the UK Chapter of the System Dynamics Society in 2007-08.

ITAMAR MEGIDDO is a Chancellor's Fellow, lecturer (assistant professor) at the Department of Management Science at the University of Strathclyde. He is also a visiting fellow at the Centre for Disease Dynamics, Economics \& Policy, Washington, DC, and he is a member of the International Decision Support Initiative. His research focus is on improving evidence-based decision making and resource allocation in healthcare policy. His main body of work integrates disease (including infection and non-communicable), health system, and economic models to evaluate impact and design policy and strategy. 